\title{
SIMULATION OF STOCHASTIC HYBRID SYSTEMS WITH SWITCHING AND REFLECTING BOUNDARIES
}

\author{
Derek Riley \\ Xenofon Koutsoukos \\ ISIS/EECS Vanderbilt University \\ Nashville, TN 37235, U.S.A.
}

\author{
Kasandra Riley \\ HHMI Yale University \\ New Haven, CT 05620, U.S.A.
}

\begin{abstract}
Modeling and simulation of biochemical systems are important tasks because they can provide insights into complicated systems where traditional experimentation is expensive or impossible. Stochastic hybrid systems are an ideal modeling paradigm for biochemical systems because they combine continuous and discrete dynamics in a stochastic framework. Simulation of these systems is difficult because of the inherent error which is introduced near the boundaries. In this work we develop a method for stochastic hybrid system simulation that explicitly considers switching and reflective boundaries. We also present a case study of the water/electrolyte balance system in humans and provide simulation results to demonstrate the usefulness of the improved simulation techniques.
\end{abstract}

\section{INTRODUCTION}

Modeling and analysis of biochemical systems are important tasks because they can provide insights into complicated systems where traditional experimentation is difficult or costly. Biological systems are often mixtures of continuous and discrete (hybrid) processes with complicated dynamics, so decoupling and studying the individual components can shed light on the function of the entire system. Biochemical processes are also inherently probabilistic because of the uncertainty of molecular motion, so models that incorporate stochasticity can provide more realistic insight into the dynamics of the system when analyzed. However, simulation of these stochastic hybrid models is a challenging task.

Simulation is a powerful analysis technique, but simulation methods must be chosen to ensure the most accurate results are generated. Simulation of systems with hybrid dynamics is especially challenging because of the error which is introduced near the switching and reflecting boundaries. A trajectory can be tested at each time step, and when it has crossed a boundary, the step is reversed and the boundary is handled. However, error is introduced because the actual trajectory can cross the boundary and return in between time steps without detection. An improvement to this method is to use probabilistic techniques to calculate the probability that the trajectory will hit the boundary in the next timestep. In this paper we develop a method for simulating Stochastic Hybrid Systems (SHS) with absorbing and reflecting boundaries that uses the boundary hit probabilities to reduce the approximation error.

To demonstrate the simulation technique, we present a case study on water/electrolyte balance in humans. All organisms must regulate the balance of water and electrolytes. In humans, this regulation is part of a system that involves the kidneys, circulatory system, pituitary gland, and other minor components. We have developed a SHS model of this intricate system to demonstrate our improved simulation methods when encountering switching and reflecting boundaries. We present the model as well as simulation results to highlight our improved boundary simulation methods.

A recently renewed interest in the field of biochemical system modeling has increased the quality and diversity of the models created. Biological protein regulatory networks have been modeled with hybrid systems using linear differential equations to describe the changes in protein concentrations and discrete switches to activate or deactivate the continuous dynamics based on protein thresholds (Ghosh and Tomlin 2004). Biomolecular network modeling using hybrid systems is accomplished by using differential equations to model feedback mechanisms and discrete switches to model changes in the underlying dynamics (Alur et al. 2001). A modeling technique that uses polynomial SHS to construct models for chemical reactions is presented in (Hespanha and Singh 2005). A SHS model of a genetic regulatory network is compared to a deterministic model in (Hu, Wu, and Sastry 2004). Switching thresholds for piecewise-affine models of genetic regulatory networks are studied in (Drulhe et al. 2006).

SHS models of biochemical systems using reaction rate analysis have been developed and simulated in (Salis and Kaznessis 2005). A biochemical system drug 
model based on physical interactions at the molecular level has been developed in (Ramos et al. 1999). An early stochastic model of the water/electrolyte balance system is presented in (Leaning et al. 1985), and a nonstochastic model with experimental results is presented in (Karanfil 2005).

Simulation of SHS is challenging because it must accurately combine numerical integration methods for Stochastic Differential Equations (SDEs) and detection/approximation of boundary crossings and reflections. Numerical integration of SDEs is accurate if the trajectory is sufficiently far from any boundaries; however, when the trajectory is close to a boundary, large errors can be incurred. A technique for accurately detecting absorbing boundaries has been developed for one-dimensional systems (Mannella 1999), and extensions have been proposed that scale to higher dimensional systems (Lamm 1983). The boundary crossing detection algorithm presented in (Peters and Barenbrug 2002) uses analysis of moments to improve the accuracy of the approximation. Methods for approximating reflecting boundaries have also been studied previously (Constantini, Pacchiarotti, and Sartoretto 1998), and an improved technique for approximating reflecting boundaries is presented in (Gobet 2001).

In this work we develop an advanced simulation technique for SHS that employs improved boundary approximation methods for absorbing and reflecting boundaries utilizing probabilistic methods. Simulation methods for SHS have been developed for the modeling language Charon, but the focus is on concurrency, and the behavior close to the boundaries is not studied (Bernadskiy, Sharykin, and Alur 2004). Improved absorbing boundary detection methods have been incorporated into a SHS simulation algorithm in (Riley, Koutsoukos, and Riley 2008). In this paper we extended the previous work by adding an improved reflecting boundary detection method and integrating it into a comprehensive SHS simulation algorithm. We also present a new case study to demonstrate the algorithm.

The rest of this paper is organized as follows: Section 2 will describe Stochastic Hybrid Systems, Section 3 will cover simulation of stochastic hybrid systems including the absorbing and reflecting boundary techniques, Section 4 will present the case study of the water balance system, and Section 5 will conclude the work.

\section{STOCHASTIC HYBRID SYSTEMS}

We use the SHS model formally defined in (Bujorianu and Lygeros 2004). To establish the notation, we let $Q$ be a set of discrete states. For each $q \in Q$, we consider the Euclidean space $\mathbb{R}^{d(q)}$ with dimension $d(q)$ and we define an invariant as an open set $X^{q} \subseteq \mathbb{R}^{d(q)}$. The hybrid state space is denoted as $S=\bigcup_{q \in Q}\{q\} \times X^{q}$. Let $\bar{S}=S \cup \partial S$ and $\partial S=\bigcup_{q \in Q}\{q\} \times \partial X^{q}$ denote the

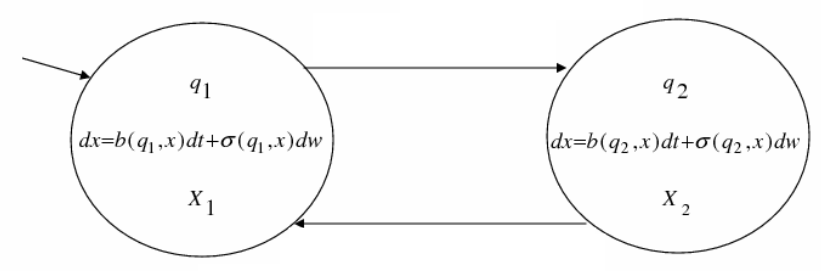

Figure 1: Stochastic hybrid system

completion and the boundary of $S$ respectively. The Borel $\sigma$-field in $S$ is denoted as $\mathscr{B}(S)$.

Consider an $\mathbb{R}^{p}$-valued Wiener process $w(t)$ and a sequence of stopping times $\left\{t_{0}=0, t_{1}, t_{2}, \ldots\right\}$. Let the state at time $t_{i}$ be $s\left(t_{i}\right)=\left(q\left(t_{i}\right), x\left(t_{i}\right)\right)$ with $x\left(t_{i}\right) \in X^{q\left(t_{i}\right)}$. While the continuous state stays in $X^{q\left(t_{i}\right)}, x(t)$ evolves according to the stochastic differential equation (SDE)

$$
d x=b(q, x) d t+\sigma(q, x) d w
$$

where the discrete state $q(t)=q\left(t_{i}\right)$ remains constant. A sample path of the stochastic process is denoted by $x_{t}(\omega), t>$ $t_{i}, \omega \in \Omega$.

The next stopping time $t_{i+1}$ represents the time when the system transitions to a new discrete state. The discrete transition occurs either because the continuous state $x$ exits the invariant $X^{q\left(t_{i}\right)}$ of the discrete state $q\left(t_{i}\right)$ (guarded transition) or based on an exponential distribution with nonnegative transition rate function $\lambda: \bar{S} \rightarrow \mathbb{R}_{+}$(probabilistic transition). At time $t_{i+1}$ the system will transition to a new discrete state and the continuous state may jump according to the transition measure $R: \bar{S} \times \mathscr{B}(\bar{S}) \rightarrow[0,1]$. The evolution of the system is then governed by the SDE (1) with $q(t)=q\left(t_{i+1}\right)$ until the next stopping time. If $t_{i+1}=\infty$, the system continues to evolve according to ( 1 ) with $q(t)=q\left(t_{i}\right)$.

Figure 1 shows a generic SHS model with two states and two transitions (one probabilistic and one guarded). The continuous dynamics of each state are defined by the associated stochastic differential equations. The probabilistic transition fires at the firing rate $\lambda$, and the guarded transition fires when $x$ hits the boundary $x \in \partial X^{q_{2}}$. The logical condition $x \in \partial X^{q_{2}}$ is often referred to as the guard of the transition. Upon firing of a transition, the state resets according to the map $R((q, x), A)$.

The following assumptions are imposed on the model. The functions $b(q, x)$ and $\sigma(q, x)$ are bounded and Lipschitz continuous in $x$ for every $q$, and thus the SDE (1) has a unique solution for every $q$. The transition rate function $\lambda$ is a bounded and measurable function which is assumed to be integrable for every $x_{t}(\omega)$. For the transition measure, it is assumed that $R(\cdot, A)$ is measurable for all $A \in \mathscr{B}(S)$ and 
$R(s, \cdot)$ is a probability measure for all $s \in \bar{S}$, and $R((q, x), d z)$ is a stochastic continuous kernel. Let $N_{t}=\sum_{i} I_{t \geq t_{i}}$ denote the number of jumps in the interval $[0, t]$. It is assumed that the expected number of jumps is finite for every initial state $s \in S$, that is $E_{S}\left[N_{t}\right]<\infty$. A sufficient condition for ensuring finitely many jumps can be formulated by imposing restrictions on $R(s, A)$ (Bernadskiy, Sharykin, and Alur 2004, Koutsoukos and Riley 2006).

\section{SHS SIMULATION}

\subsection{Background}

Simulating SHS is important because it can shed light on complicated models and dynamics. However, it is challenging because the interplay between the stochastic continuous and discrete dynamics can cause large errors if handled incorrectly. Switching and reflecting boundaries must be detected precisely to avoid incurring error of assuming false crossings or missing real crossings. The error can be decreased by reducing the step size of the approximation, but this comes at the cost of efficiency. Improved boundary simulation techniques are able to take better advantage of smaller step sizes using probabilistic sampling, but they require more involved calculations which can decrease efficiency.

Numerical integration methods for SDEs assume that the solution is sufficiently far from any boundaries; however, this assumption does not hold for SHS where the effect of the switching and reflective boundaries must be taken into account. Large errors can be incurred if the boundary conditions are not handled carefully. Let us assume a system has an invariant $X^{q}$ with a boundary $\partial X^{q}$, and the state at time $t$ is $X(t)$. In the case of switching boundaries, as seen in Figure 2, it is possible that $X(t), X(t+\Delta t) \in X^{q}$, but $\exists \tau \in[t, t+\Delta t]$ where $x(\tau) \notin X^{q}$. In this case, a boundary crossing occurs in the actual execution of the SHS but not in the approximating solution, and this discrepancy may cause a significant error.

Reflecting boundaries are difficult to approximate because the crossing must be detected, and the reflection must be calculated without incurring large errors. Approximating the crossing of the boundary incurs similar error to approximating the crossing of the switching boundary. Error generated in the approximation of the crossing is amplified when calculating the reflection, so care must be taken when approximating the crossing. The traditional way to reflect the process is to detect the first crossing of the boundary and reverse the dynamics to force the system back into the valid region. This type of reflection may not be appropriate for the process, and care must be taken to ensure that the process is reflected into a valid state. Therefore, improved methods are necessary to ensure the crossing and reflection are calculated accurately.

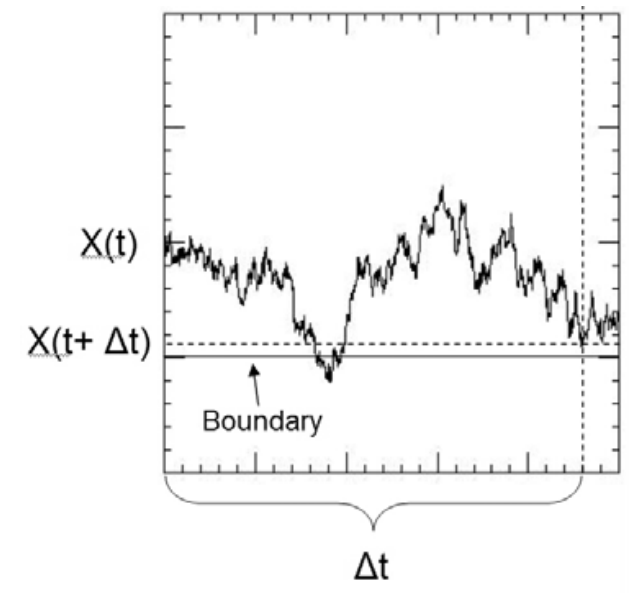

Figure 2: A SHS trajectory close to a boundary

The error introduced by the various approximation methods is quantified by the concept of order of convergence and defines the quality of the approximation for stochastic simulation techniques. An approximation $X^{\Delta t}(T)$ at time $T$ with step size $\Delta t$ converges with order $\gamma$ strongly to the actual trajectory $x(T)$ if there exists $c>0$ such that $E\left(\left|x(T)-X^{\Delta t}(T)\right|\right) \leq c \Delta t^{\gamma} . X^{\Delta t}(T)$ converges with order $\gamma$ weakly to $x(T)$ if there exists $c>0$ such that $E\left(\left|f(x(T))-f\left(X^{\Delta t}(T)\right)\right|\right) \leq c \Delta t^{\gamma}$ for a given class of measurable functions $f$ (Kloeden and Platen 1999). Strong convergence implies that the trajectory is a possible trajectory of the system, and weak convergence implies that the computed trajectory only preserves the moments of the actual trajectory.

\subsection{Simulation of SDEs}

Simulation of SDEs can be performed using Taylor schemes of various orders. The simplest Taylor approximation scheme is the Euler Maruyama (EM) method which is a first-order approximation. The $k$ th component of the EM scheme is given by

$$
X_{n+1}^{k}=X_{n}^{k}+b^{k} \Delta t+\sum_{j=1}^{m} \sigma^{k, j} \Delta W^{j}
$$

for $k=1,2, \ldots, d$ where $\Delta W^{j}$ is the normally-distributed increment of the $j$ th component of the $d$-dimensional Wiener process $W$ assuming a $d$-dimensional drift coefficient $b$ and a $d \times m$ diffusion coefficient $\sigma$.

The Milstein Method (MM) is a second-order Taylor scheme. The higher order terms require more computation; however, the approximation maintains an acceptable efficiency for most systems. It extends the EM method by adding terms to the approximation; however, if the diffu- 
sion coefficient $\sigma$ is constant, all higher order approximation terms are zero (Kloeden and Platen 1999). The diffusion term $\sigma$ in our case study is constant, so we only present the EM method in this work.

Taylor schemes can have strong order of convergence of $\gamma=0.5$ to $\gamma=3.0$ and weak order of convergence of $\gamma=1.0$ to $\gamma=6.0$ depending on the number of approximating terms (Kloeden and Platen 1999). The computation of higher order terms requires many more operations and can be prohibitively complicated and expensive; therefore, a tradeoff must be reached to achieve the appropriate accuracy and efficiency. The EM method is simple to implement, but achieves a strong convergence of $\gamma=0.5$ and weak convergence $\gamma=1.0$, whereas the MM has a strong order of convergence of $\gamma=1.0$.

\subsection{Switching Boundaries}

During the execution of a SHS, the process can hit a switching boundary defined by the invariants. At a switching boundary the continuous process is halted and re-started in a new state after executing any transition resets. Switching boundaries can therefore be treated as absorbing boundaries. It is important to accurately estimate the time and location that the process is absorbed to minimize the error introduced into the approximation.

The easiest way to detect an absorbing boundary is to check the state against the invariants at each step of the approximation. Let us assume the state at time $t$ is $X(t)$. If $X(t) \in X^{q}$, but $X(t+\Delta t) \notin X^{q}$, then the process is rolled back to time $t$ and re-started in the new state. This method has a strong order of convergence of $\gamma=0.5$ (Peters and Barenbrug 2002).

An improved method for absorbing boundary detection based on probabilistic sampling was developed in (Gobet 2001). The approach can be used with boundaries that are hyperplanes or sufficiently smooth. The biochemical models we consider have boundaries that are hyperplanes, so this approach is valid for these systems. The probability that the state trajectory has hit the boundary between $t$ and $t+\Delta t$ is

$$
P(h i t)=\exp \left(\frac{-2\left(n \cdot\left(X_{t}-X_{a b}\right)\right)\left(n \cdot\left(X_{t+\Delta t}-X_{a b}\right)\right)}{n \cdot\left(\sigma\left(X_{t}\right) \sigma^{*}\left(X_{t}\right) n\right) \Delta t}\right)
$$

where the switching boundaries are hyperplanes $\partial X^{q}=$ $\left\{x \in \mathbb{R}^{d(q)}: n \cdot\left(x-X_{a b}\right)=0\right\}, n$ is the unit vector normal to the boundary $\partial X^{q}, X_{a b} \in \mathbb{R}^{n}$ is the position of the absorbing boundary, and $X_{t}$ is the computed continuous state at time $t$. For simplifying the notation we have chosen to describe the diffusion $\sigma\left(X_{t}\right)$ as only depending on the continuous state, but the actual diffusion may depend on the discrete state as well. This improved method achieves a weak order of

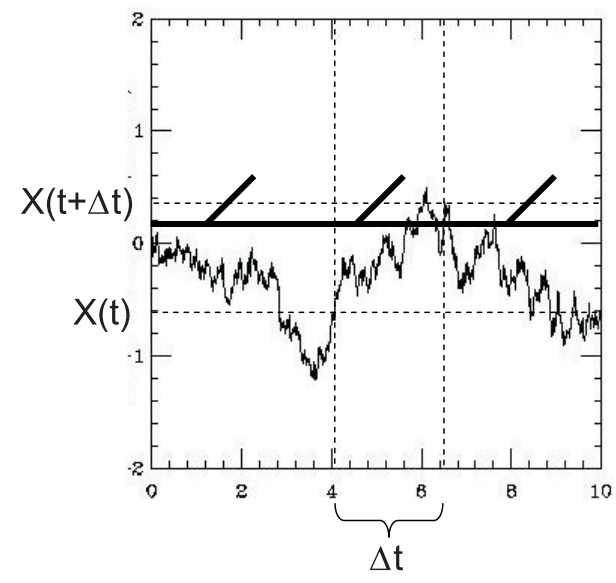

Figure 3: Boundary reflection problem

$\gamma=1.0$ assuming that the boundary is sufficiently smooth (Gobet 2001).

\subsection{Reflective Boundaries}

Invariants may include reflective boundaries in addition to the switching boundaries. These invariants can cause the process to be reflected obliquely when it reaches the boundary. For example, all biochemical systems cannot contain negative concentrations of any chemicals, or some biochemical processes also have saturation limits which impose upper limits on concentrations. In both cases when the process reaches the boundary, it should be reflected to mimic the behavior of the real world system.

The traditional way to handle reflective boundaries is to detect them like absorbing boundaries and reflect the dynamics when the process leaves the valid state space defined by the invariants (Gobet 2001). Let us assume a system has an invariant $X^{q}$ with a reflective boundary, and the state at time $t$ is $X(t)$. If $X(t) \in X^{q}$, but $X(t+\Delta t)$ is computed to be outside of $X^{q}$, then the process is reflected in the direction normal to the boundary $X(t+\Delta t) . n=X(t) . n$ where $n$ is the unit vector normal to the boundary. In Figure 3 the reflected boundary is detected at $t+\Delta t$ but the actual trajectory should have been reflected before then, so the trajectory is rolled back to time $t$. This method has a weak order of convergence of $\gamma=0.5$ (Gobet 2001).

The improved method described in (Gobet 2001) defines a new diffusion process which adds the effect of the reflection to the original $\mathrm{SDE}$ :

$$
d x=b(q, x) d t+\sigma(q, x) d w+n(q, x) d k
$$




\section{Riley, Koutsoukos, and Riley}

where $n(q, x)$ is a unit vector normal the boundary at state $(q, x), k=\int_{0}^{t} 1_{X \in \partial D} d k, \partial D$ is the reflective boundary, and $X_{r b}$ is the position of the reflective boundary.

The approximation of the process is calculated using:

$$
X_{t+\Delta t}=X_{t}+b \Delta t+\sigma \Delta W+n \Delta k
$$

where $\Delta W$ is a normally-distributed pseudo-random number and $\Delta k=k_{t}-k_{t+\Delta t}$. Approximating $k_{t}$ is achieved using the technique described in (Gobet 2001):

$k_{t}=\max \left(0, z_{t}\right) \cdot n$
$z_{t}=X_{t}-X_{r b}+\frac{1}{2}\left(\sigma W+b t+\sqrt{|\sigma|^{2} V+(\sigma W+b t)^{2}}\right)$

where $V=\varepsilon(1 / 2 t)$ is an exponentially-distributed random variable independent of $W$. This equation is derived from the solution to the Skorohod problem and results in a weak order 1.0 approximation of the reflecting boundary (Gobet 2001).

\subsection{SHS Simulation Algorithm}

Simulation of SHS requires the combination of simulation methods for SDEs, switching boundaries, reflecting boundaries, and probabilistic transitions. We have implemented the improved absorbing boundary detection method and incorporated it into the EM method to improve the SHS simulation algorithm. At each time step we calculate the probability of hitting all nearby boundaries $P($ hit $)$. We then select the boundary with the highest hitting probability and compare the probability to a uniformly-distributed number $U_{1}$. When $U_{1}<P($ hit $)$, then we consider the boundary to be hit, and we execute the transition resets and restart the process in the new state.

We have also incorporated the improved reflecting boundary method into the SHS simulation algorithm. At each step we begin by storing the previous $\Delta k$ value and calculating the new $\Delta k$. If the trajectory is close to a reflecting boundary, we add $\Delta k$ to the EM computation according to the method described earlier.

To simulate probabilistic transitions, we use the technique described in (Bernadskiy, Sharykin, and Alur 2004). We begin by computing the hitting probability at each step using the following equation $P($ hit $)=\exp (-\lambda(t-$ $\left.t_{\text {TimeOfLastFire }}\right)$ ). We then compare $P($ hit $)$ to a uniformlydistributed number $U_{2}$, and when $U_{2}<P($ hit $)$ we assume the transition has fired. We initially assume the $t_{\text {TimeOfLastFire }}=0$, and we update it each time the transition fires. The following algorithm describes a single step of the combined iterative technique.

\section{Algorithm 3.1: SHSSimulationSteP $\left(X_{t}^{k}, \operatorname{SimLength}\right)$}

$$
\begin{aligned}
& k_{t+\Delta t}=X_{t}-X_{r b}+ \\
& +\frac{1}{2}\left(\sigma W+b(t+\Delta t)+\sqrt{|\sigma|^{2} V+(\sigma W+b(t+\Delta t))^{2}}\right) \\
& \Delta k=\max \left(k_{t+\Delta t}, 0\right) \cdot n-\operatorname{prev} \Delta k \\
& X_{t+\Delta t}^{N}=X_{t}^{N}+b^{N}(t+\Delta t)+\sigma^{N} \Delta W+n(\Delta k) \\
& t++ \\
& \text { if } U_{1}=\operatorname{rand}(0,1)<\exp \left(\frac{-2\left(n .\left(X_{t}^{k}-X_{a b}\right)\right)\left(n .\left(X_{t+\Delta t}^{k}-X_{a b}\right)\right)}{n \cdot\left(\sigma \sigma^{*}\left(X_{t}\right) n\right) \Delta t}\right) \\
& \text { then FireGuardedTransition } \\
& \text { if } U_{2}=\text { rand }(0,1)<\exp \left(-\lambda\left(t-t_{\text {TimeOfLastFire }}\right)\right) \\
& \text { then FireProbabilisticTransition }
\end{aligned}
$$

The approximations using the EM method, boundary methods, and probabilistic transitions converge to the actual solution individually as the step size is decreased to zero, so their combination will also converge to the correct solution. By combining methods with higher order convergence, we reduce approximation error more quickly than the lower order methods therefore improving efficiency and accuracy. The traditional absorbing and reflecting boundary algorithms have a weak order of convergence of $\gamma=0.5$, while the improved methods both have a weak order of convergence of $\gamma=1.0$ (Gobet 2001). The combined SHS algorithm we present therefore has a weak order of $\gamma=1.0$ since the EM has a weak order of convergence $\gamma=1.0$ and the other two included methods also have a weak order of convergence of $\gamma=1.0$. The MM method can be used to improve the SDE approximation as well, but is not necessary for the case study in this paper.

Error is introduced into the calculated SHS trajectory in several different ways. Approximation of the SDE introduces higher-order errors which are not calculated due to computational inefficiency. Error due to the use of pseudorandom numbers is typically not a concern for smaller simulations, but large simulations or Monte Carlo methods must use pseudo-random generators which do not repeat as often as the efficient generators to avoid this type of error. Finally, step size inherently introduces error in the SDE and boundary calculations as described earlier by the order of convergence $\gamma$.

Accurate simulation of the trajectory near intersections of boundaries is a difficult problem, and must be handled carefully to minimize error. When the trajectory is in close proximity to multiple reflecting or absorbing boundaries, our algorithm considers the boundary with the highest hitting probability at each time step. 


\section{CASE STUDY: Water Balance}

\subsection{Background}

Water/electrolyte balance regulation in mammals is vital to life. If too much salt is present, dehydration occurs, leading to discomfort, performance degradation, and even death. If too much water is present, arterial pressure rises dangerously and the nervous system begins to malfunction. Therefore, virtually every living organism has a system that regulates water balance. In humans, this system includes blood pressure sensors, the kidneys, the hypothalamus, and other minor organs.

Anti-diuretic hormone (ADH) is a nine amino acid peptide hormone secreted by the hypothalamus. ADH is released when the body senses the intake of too much salt or a shortage of water. Upon these conditions ADH signals to the kidneys to retain water to compensate and bring the body back to equilibrium. Upon secretion, ADH travels through the bloodstream to exert the majority of its effects on specific receptors (arginine vasopressin receptor 2; AVPR2) in specialized cells within the kidney tubules. When ADH binds AVPR2, a chain of intracellular signaling events take place. The succession of signaling events ultimately results in additional insertion of extra water channels (aquaporins;AQP2) into the apical membrane of the cell. Aquaporins allow water to pass out of the nephrons and be re-collected into the cells. Once the water is reabsorbed, a smaller, more concentrated amount of urine is excreted.

The insertion of AQP2 channels into the cell's outer membrane is a highly regulated, multistep process. AQP2 is synthesized in the cell and inserted into intracellular membrane structures called vesicles. When called upon by ADH-AVPR2 interaction and resulting intracellular signaling, attachment and tethering proteins specifically direct the vesicles to fuse with the outer membrane of the cell. The fusion event results in the addition of the AQP2 molecules to the outer membrane. The total number of available AQP2-containing vesicles and the attachment and tethering proteins are both inherently limited in any given cell resulting in a saturation point for sensitivity of the cell to ADH (Noda and Sasaki 2006).

When ADH is withdrawn, AQP2 accumulates in special membrane domains (clathrin-coated pits), which are subsequently engulfed (endocytosed) by the cell. Endocytosed AQP2 receptors are then recycled within the cells, ready for the next ADH signal. AQP2 is continuously and quickly recycled between the cell surface and intracellular compartments, rebounding between upper and lower limits for AQP2 cell surface localization. This behavior results in a reflection of the observed effects at the ADH saturation limit (Noda and Sasaki 2006).

\subsection{SHS Model}

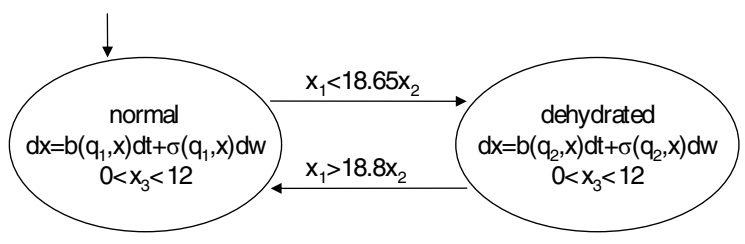

Figure 4: SHS model of the water balance model

We have developed a SHS model of the water/electrolyte balance system, seen in Figure 4. The SHS has been adapted from the SDE model in (Leaning et al. 1985) to include the hybrid thirst/dehydration mechanism described in (Karanfil 2005). The model includes two discrete states: normal and dehydrated. Transitions between the normal and dehydrated modes are defined by the transition guards in Figure 4 and are based on the ratio of water to salt (or electrolyte concentration) in the body derived from data in (Karanfil 2005).

We define three continuous states: total body water $x_{1}$, total body salt $x_{2}$, and $\mathrm{ADH} x_{3}$ within each discrete state. The dynamics for the water and salt variables were based on simple input/output differences in the system with an added diffusion term that models uncertainty and system variability (Leaning et al. 1985). SDEs are used with constant diffusion because of the uncertainty of molecular interactions in these types of biochemical systems. Fluid output is directly dependent on the ADH concentration which is in turn affected by the fluid/salt ratio in the body.

The following SDEs desribe the continuous dynamics in the normal state

$$
\begin{aligned}
& d x_{1}=\left(f_{\text {in }}-45 x_{3}^{-.76}\right) d t+.1 d w_{1} \\
& d x_{2}=\left(s_{\text {in }}-s_{\text {out }}\right) d t+.1 d w_{2} \\
& d x_{3}=(-4.5) d t+.1 d w_{3}
\end{aligned}
$$

where $f_{\text {in }}$ describes the amount of fluid input to the system per unit time, $s_{i n}$ describes the amount of salt input to the system per unit time, $s_{\text {out }}$ describes the amount of salt output from the system per unit time, and $w=\left[w_{1}, w_{2}, w_{3}\right]^{T}$ is a three-dimensional Wiener process.

The next set of equations describe the dynamics when the body is in the dehydrated state determined by the electrolyte concentration. 
Table 1: Model Coefficients

\begin{tabular}{|c|c|}
\hline Variable & Value \\
\hline$f_{\text {in }}$ & 40 \\
\hline$s_{\text {in }}$ & 2 \\
\hline$s_{\text {out }}$ & 2 \\
\hline
\end{tabular}

$$
\begin{aligned}
& d x_{1}=\left(f_{\text {in }}-45 x_{3}^{-.76}\right) d t+.1 d w_{1} \\
& d x_{2}=\left(s_{\text {in }}-s_{\text {out }}\right) d t+.1 d w_{2} \\
& d x_{3}=\left(\frac{80 * x_{2}}{x_{1}}\right) d t+.1 d w_{3}
\end{aligned}
$$

The constants for the continuous dynamics were adapted from (Leaning et al. 1985) to match the experimental data in (Karanfil 2005). We fit the experimental data to curves and determined appropriate adaptations for the dynamics when necessary. The values we used for our experiments can be found in Table 1 . The fluid input $f_{\text {in }}$ can be modeled as a continuous stream or discrete input, so for simplicity we consider only the continuous stream. Since our focus was primarily on the water balance, we modeled $s_{\text {in }}$ and $s_{\text {out }}$ as constant functions; however, these could be easily extended to model more realistic behavior if salt balance is the focus of the analysis.

Because ADH cannot have a negative value a reflective boundary is defined for $x_{3}$ at the value of zero. We also define a reflective boundary at $x_{3}=12$ to mimic the saturation limit of ADH in the kidneys. The limit is defined by the invariants in the system $x_{3} \in 0,12$. This range will not necessarily be the same for every person, but seems reasonable based on experimental data from (Karanfil 2005).

Simulation of this model is important because it may help improve the understanding of the biological system and identify statistically significant aberrations in patients. Efficient, accurate simulation techniques are important to be able to refine the model and perform Monte Carlo analysis on the simulation data.

\subsection{Experimental Results}

We implemented the SHS simulation algorithm presented in Section 3. To evaluate the performance of the algorithm we use as a baseline a simple method that detects boundary crossings at each time step and either rolls back (switching boundaries) or reflects the state (reflecting boundaries) as described in Section 3. We used the same Brownian motion for each set of simulations to highlight the differences. In Figure 5 the switching electrolyte boundary is presented for the traditional and improved methods, and the difference

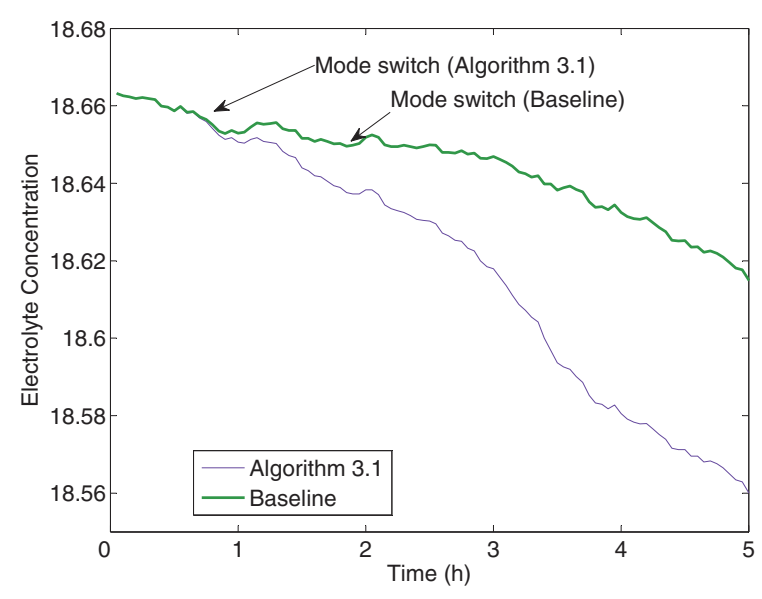

Figure 5: Absorbing boundary in water balance model

between the detection times is shown by the gap between the indicated detection points. The proposed method anticipates the boundary crossing using probabilistic methods to avoid error incurred by over-shooting the crossing, while the baseline method re-starts the process only after the crossing is detected. It is evident that the anticipatory methods of the improved technique significantly alter the resulting trajectory thereby reducing the error incurred. In the water/electrolyte system this may mean that the actual system will react sooner than the model will indicate with the traditional simulation method predicts. For these simulations we used a step size of $\Delta t=.05$ and initial conditions shown in Table 2.

We consider the ADH concentration to demonstrate the reflecting boundary algorithm differences. In Figure 6 the reflecting boundary is represented by the dark line at $A D H=12$. In the baseline method, the trajectory reaches the boundary and is kept within the valid state. However, the dynamics of the actual system are not accurately represented because the real system reaches a reflecting saturation level at the boundary. In out method, the trajectory highlights the probabilistic effect of the reflected saturation boundary. The receptors in the real system cannot maintain the full concentration at 12 because the molecules of $A D H$ have to be released to permit new molecules to bind. The receptors cannot fire in an unbound state, so the influence of the $A D H$ concentration must be reduced, as is evidenced by the small drops in concentration near the boundary. These drops eventually lead to a distinct difference between the outcomes of the two trajectories. While both trajectories eventually reach an equilibrium (not included in the figure), the difference in the dynamics leading to equilibrium may reveal new insights into the system. For these simulations, we used a step size of $\Delta t=.05$ and initial conditions shown in Table 2. 


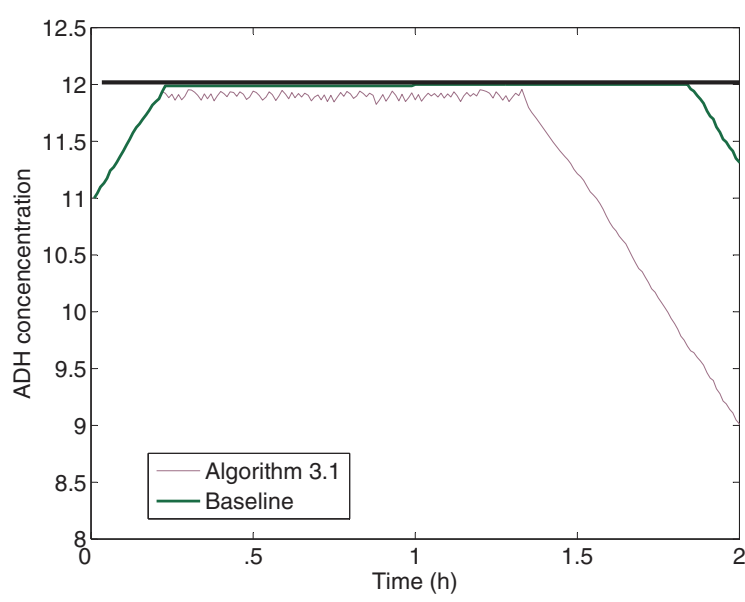

Figure 6: Reflecting boundary in the water balance model

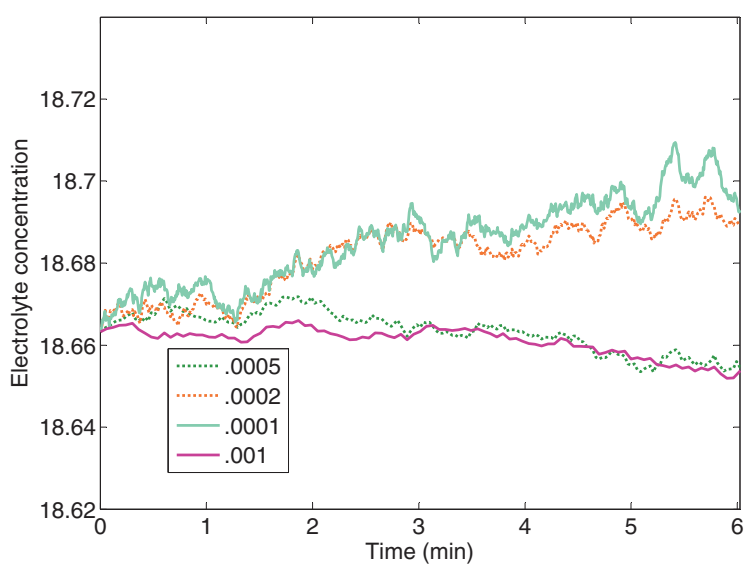

Figure 7: Step size comparison

The step size of the approximation directly influences the accuracy of the approximation. In Figure 7, we compare four different step sizes and resulting trajectories with the same initial conditions as the absorbing boundary example. We saved the Brownian motion from the highest-resolution trajectory to match it at each lower-resolution time step to ensure the comparison is appropriate. At very fine resolutions the system becomes highly noisy. Thus, using more accurate approximation techniques with higher orders of convergence ensures that larger time steps can be used to maintain acceptable accuracy without having to approximate the highly noisy dynamics that lead to zeno behavior at boundaries if not handled carefully.

The performance results are presented in Table 3. We ran 1000 sequential simulations of each algorithm at the given resolution. The proposed method increases the running time relative to the baseline method; however, the increase is small, the method scales well, and the accuracy improvement
Table 2: Initial conditions

\begin{tabular}{|c|c|c|c|}
\hline Variable & $x_{1}$ & $x_{2}$ & $x_{3}$ \\
\hline Absorbing & 39790 & 2132 & 1 \\
\hline Reflecting & 39700 & 2132 & 11 \\
\hline
\end{tabular}

Table 3: Execution times (sec)

\begin{tabular}{|c|c|c|}
\hline Resolution & Baseline & Algorithm 3.1 \\
\hline .0001 & 352 & 374 \\
\hline .0002 & 176 & 189 \\
\hline .0005 & 70 & 75 \\
\hline .001 & 37 & 38 \\
\hline
\end{tabular}

is significant. The simulations were performed on a $3 \mathrm{GHz}$ desktop computer with $1 \mathrm{~GB}$ of RAM.

\section{CONCLUSION}

Accurate and efficient simulation of SHS is an important task because it is an important tool that can expose the intricacies of the complicated dynamics of highly-coupled systems like biochemical processes. The interplay between the continuous and discrete dynamics in SHS can introduce large errors into the simulations at the boundaries, so they must be approximated carefully. The EM technique for simulating SDEs combined with probabilistic absorbing boundary detection and reflecting boundary calculation improves the accuracy and efficiency of the simulator when compared with the naive approaches.

\section{REFERENCES}

Alur, R., C. Belta, F. Ivanifi, V. Kumar, M. Mintz, H. Rubin, and J. Schug. 2001. Hybrid modeling and simulation of biomolecular networks. In Hybrid Systems Computation and Control, Volume LNCS 2034, 19-33.

Bernadskiy, M., R. Sharykin, and R. Alur. 2004. Structured modeling of concurrent stochastic hybrid systems. FORMATS LNCS 3253:309-24.

Bujorianu, M., and J. Lygeros. 2004. Theoretical foundations of general stochastic hybrid systems: Modeling and optimal control. In IEEE Conf. on Dec. and Cont.

Constantini, C., B. Pacchiarotti, and F. Sartoretto. 1998. Numerical approximation for functionals of reflecting diffusion processes. SIAM J. Appl. Math 58:73-102.

Drulhe, S., G. Ferrari-Trecate, H. de Jong, and A. Viari. 2006. Reconstruction of switching thresholds in piecewise-affine models of genetic regulatory networks. 


\section{Riley, Koutsoukos, and Riley}

In Hybrid Systems Computation and Control, Volume LNCS 3927, 184-99.

Ghosh, R., and C. Tomlin. 2004. Symbolic reachable set computation of piecewise affine hybrid automata and its application to biological modeling: Delta-notch protein signalling. Sys. Bio. 1:170-83.

Gobet, E. 2001. Euler schemes and half-space approximation for the simulation of diffusion in a domain. ESAIM: Probability and Statistics 5:261-97.

Hespanha, J., and A. Singh. 2005. Stochastic models for chemically reacting systems using polynomial stochastic hybrid systems. Int. J. on Robust Cont., Special Issue on Control at Small Scales 15:669-89.

$\mathrm{Hu}$, J., W. Wu, and S. Sastry. 2004. Modeling subtilin production in bacillus subtilis using stochastic hybrid systems. In Hybrid Systems Computation and Control, Volume LNCS 2993, 417-31.

Karanfil, O. 2005. A dynamic simulator for the management of disorders of the body water metabolism. Master's thesis, Bogazici University.

Kloeden, P., and E. Platen. 1999. Numerical solution of stochastic differential equations. Springer-Verlag.

Koutsoukos, X., and D. Riley. 2006. Computational methods for reachability analysis of stochastic hybrid systems. In Hybrid Systems Computation and Control, Volume LNCS 3927, 377-91.

Lamm, G. 1983. Extended brownian dynamics. iii. three dimensional diffusion. J. Chem. Phys. 80 (6): 2845-55.

Leaning, M., R. Flood, D. Cramp, and E. Carson. 1985. A system of models for fluid-electrolyte dynamics. IEEE Trans. on Biomed. Eng. 32 (10): 856-64.

Mannella, R. 1999. Absorbing boundaries and optimal stopping in a stochastic differential equation. Phys. Lett. A 254:257-62.

Noda, Y., and S. Sasaki. 2006. Regulation of aquaporin-2 trafficking and its binding protein complex. Biophysica Acta 1758:1117-1125.

Peters, E., and T. Barenbrug. 2002. Efficient brownian dynamics simulation of particles near walls. i. reflecting and absorbing walls. Physical Review 66:1-7.

Ramos, M., A. Melo, E. Henriques, J. Gomes, N. Reuter, B. Maigret, W. Floriano, and M. Nascimento. 1999. Modeling enzyme-inhibitor interactions in serine proteases. Int. J. Quant. Chem. 74 (3): 299-314.

Riley, D., X. Koutsoukos, and K. Riley. 2008. Modeling and simulation of biochemical processes using stochastic hybrid systems: The sugar cataract development process. In Hybrid Systems Computation and Control LNCS 4981, 429-42.

Salis, H., and Y. Kaznessis. 2005. Accurate hybrid stochastic simulation of a system of coupled chemical or biochemical reactions. J. Chem. Phys. 122:54-103.

\section{AUTHOR BIOGRAPHIES}

DEREK D. RILEY received B.A. degrees in computer science and math from Wartburg College, Waverly, IA, in 2004, and the M.S. degree in computer science from Vanderbilt University, Nashville, TN, in 2006. Since June 2005, he has been a Graduate Research Assistant with the Institute for Software Integrated Systems and Department of Electrical Engineering and Computer Science, Vanderbilt University, Nashville, TN, working towards the Ph.D. degree in computer science. His email address for these proceedings is <derek.riley@vanderbilt.edu>.

XENOFON KOUTSOUKOS received his Diploma in Electrical and Computer Engineering from the National Technical University of Athens (NTUA), Greece in 1993, M.S. degrees in Electrical Engineering and Applied Mathematics in 1998, and his Ph.D. in Electrical Engineering in 2000 from the University of Notre Dame. Since 2002, he has been with the Department of Electrical Engineering and Computer Science at Vanderbilt University where he is currently an Assistant Professor and a Senior Research Scientist in the Institute for Software Integrated Systems (ISIS). His email address for these proceedings is <xenofon. koutsoukos@vanderbilt.edu>.

KASANDRA J-L. RILEY received B.A. degrees in Chemistry, Biochemistry, and Biology from Wartburg College, Waverly, IA, in 2002, and a Ph.D. in Biomedical Science: Biochemistry and Molecular Biology from Mayo Clinic College of Medicine, Rochester, MN, in 2007. She is currently a postdoctoral research fellow with Dr. Joan Steitz in the Molecular Biophysics and Biochemistry Department at Yale University (Howard Hughes Medical Institute). Her email address for these proceedings is <kasandra.riley@yale.edu>. 